Case Report

\title{
Ocular Rosacea Causing Corneal Melt in an African American Patient and a Hispanic Patient
}

\author{
Joanna S. Saade, ${ }^{1}$ Bachir Abiad, ${ }^{1}$ Jonathan Jan, ${ }^{1}$ Dana Saadeh, \\ James P. McCulley, ${ }^{1}$ and Jeremy Bartley ${ }^{1}$ \\ ${ }^{1}$ University of Texas Southwestern Medical Center, Ophthalmology Department, Dallas, TX, USA \\ ${ }^{2}$ American University of Beirut Medical Center, Dermatology Department, Beirut, Lebanon \\ Correspondence should be addressed to Joanna S. Saade; joannasaadeh@gmail.com
}

Received 27 April 2017; Accepted 10 September 2017; Published 11 October 2017

Academic Editor: Huban Atilla

Copyright (C) 2017 Joanna S. Saade et al. This is an open access article distributed under the Creative Commons Attribution License, which permits unrestricted use, distribution, and reproduction in any medium, provided the original work is properly cited.

\begin{abstract}
Purpose. To discuss two rare presentations of ocular rosacea in a Hispanic patient and an African American patient with unusual ocular manifestations. Case Report. Case 1: a 43-year-old Hispanic woman presented with right eye corneal perforation. Her prior medical history was significant for rosacea only, diagnosed clinically by a dermatologist. Her eye exam showed signs of bilateral ocular rosacea. An emergent full thickness tectonic corneal patch graft was done. The patient's bilateral eye symptoms improved one month after initiating rosacea treatment. Case 2: a 51-year-old African American man with long standing history of untreated rosacea presented with bilateral peripheral corneal thinning with neovascularization that led to right eye corneal perforation. Glue and bandage contact lens were applied. The patient did well 4 weeks after starting antibacterial, oral steroids, and rosacea treatment. Discussion. Ocular rosacea can present in Hispanic and African American patients with severe manifestations such as corneal perforation.
\end{abstract}

\section{Rosacea}

Rosacea is a chronic inflammatory cutaneous condition with a fluctuating course. It is characterized by flushing, nontransient erythema, papules, pustules, and telangiectasias. It is most commonly described in the white population of western and northern descent [1-3]. Although underestimated, it is also present in people with skin of color $[4,5]$. Some reports state that $4 \%$ of rosacea patients are African American, Hispanic, or Asian $[6,7]$. One study has shown that $2 \%$ of patients diagnosed with rosacea are African American and $3.5 \%$ are Hispanic [8].

Ocular manifestations have been reported in $3-58 \%$ of patients with rosacea [9-11]. They range from mild blepharoconjunctivitis with injection, tearing, and burning sensation to sight-threatening corneal neovascularization, scarring, thinning, and perforation [12].

Ocular rosacea was first described in African American patients in 1986 [13]. To our knowledge, there has not been a reported case of a Hispanic patient with corneal perforation secondary to rosacea. We present two cases of corneal perforation in patients with skin of color.

\section{Case Report}

2.1. Case 1. A 43-year-old Hispanic woman presented with recurrent asymmetrical bilateral eye redness, tearing, and pain for the past 2 years, with more severe symptoms in the right eye. Her ocular history was negative prior to this presentation. Her past medical history is significant only for rosacea, diagnosed clinically more than ten years ago by a dermatologist. She denied illicit drug use, exposure to patients with tuberculosis, or promiscuous sexual activity. Her family history is negative for ocular diseases and for autoimmune diseases. Aside from her eyes and her skin complaints, her review of systems was negative. Her right eye visual acuity was 20/40 and intraocular pressure was $8 \mathrm{~mm} \mathrm{Hg}$. Right eye slit lamp exam showed iris plugging a perforated cornea superonasally with associated extensive 


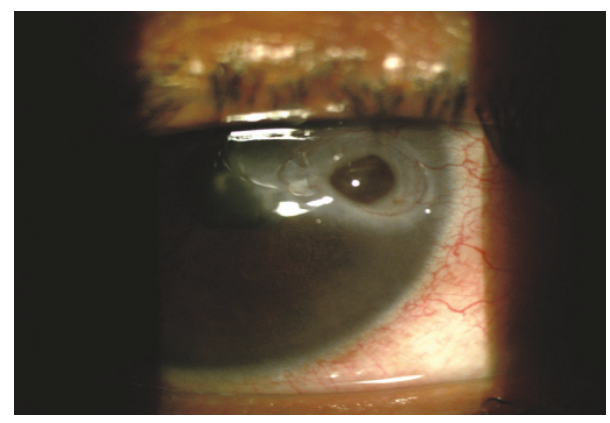

FIGURE 1: Slit lamp photo of the right eye showing the iris plugging the corneal perforation.

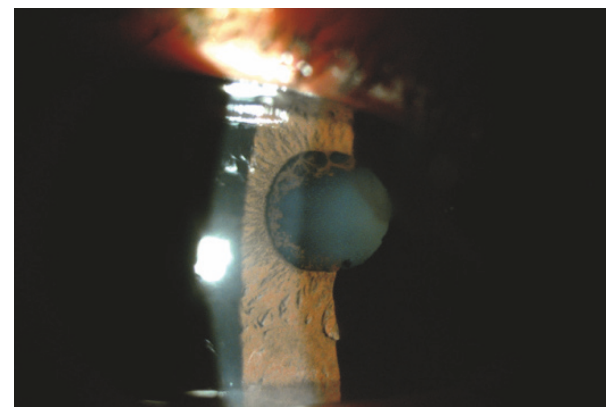

FIGURE 2: Slit lamp photo of the right eye showing a formed anterior chamber.

neovascularization (Figure 1) and a shallow but formed anterior chamber (Figure 2).

The cornea in her left eye appeared normal. Signs of bilateral blepharitis due to ocular rosacea such as eyelid telangiectasias, thick meibomian gland secretions with digital compression, and collarettes on the base of upper and lower eyelashes were present. The patient consented to an emergent, full thickness, tectonic corneal patch graft. Postoperative management consisted of topical steroid (prednisolone acetate ophthalmic solution 1\%) every two hours that was tapered slowly after one-week period and topical moxifloxacin four times daily for a total period of two weeks. Concurrently a dermatologist initiated rosacea treatment consisting of minocycline $100 \mathrm{mg}$ orally twice daily and benzoyl peroxide/erythromycin gel daily. At week one after grafting, the visual acuity was 20/100 (Figure 3).

The patient's bilateral eye signs and symptoms improved significantly one month after initiating rosacea treatment.

2.2. Case 2. A 51-year-old African American man presented with bilateral eye pain and redness of four months' duration. Patient denied ocular problems previously. His prior medical history was significant for rosacea, for which he was not receiving any medical treatment. His review of systems was positive for bilateral eye pain and tender facial papules and pustules. He denied family history of autoimmune diseases or eye diseases. He denied promiscuous sexual activity, exposure to tuberculosis, or illicit drug use. His external exam

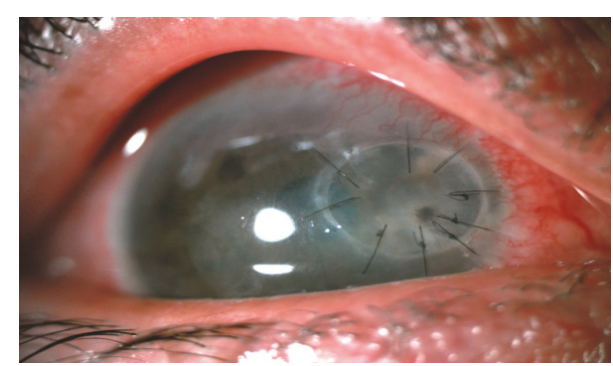

FIGURE 3: Slit lamp photo of the right eye showing a full thickness corneal patch graft, 1 week following the surgery.

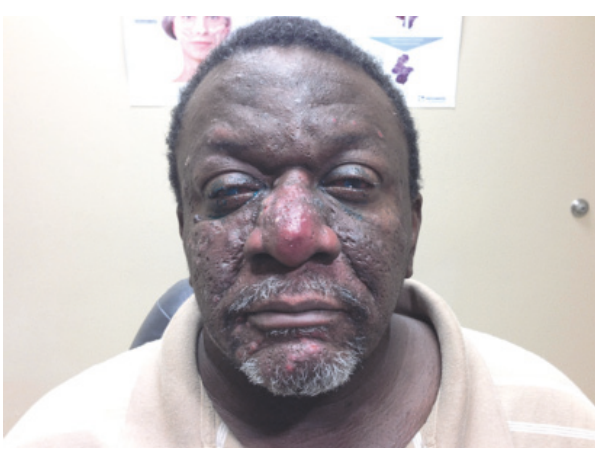

FIGURE 4: Rosacea manifesting with papules and pustules affecting the patient's face.

was consistent with multiple medium to large erythematous papules, pustules with rhinophyma (Figure 4).

A skin biopsy was done and it revealed disrupted follicles with surrounding mixed suppurative and granulomatous dermatitis most suggestive of acneiform processes including ruptured folliculitis and rosacea. The patient was started on doxycycline $100 \mathrm{mg}$ twice daily and topical metronidazole $1 \%$ cream twice daily.

His visual acuity was 20/400 in the right eye and 20/30 in the left eye. Slit lamp exam showed severe bilateral conjunctival injection, corneal pannus associated with inferonasal peripheral corneal thinning, and stromal scarring. His right eye showed an inferior $3 \mathrm{~mm}$ light-blocking infiltrate with overlying $3 \mathrm{~mm}$ epithelial defect.

Peripheral ulcerative keratitis (PUK) was suspected and oral prednisone $60 \mathrm{mg}$ was started. An extensive autoimmune workup was done. All results were negative. Cornea was scraped for gram stain, bacterial cultures, and fungal cultures. The patient was started on topical hourly fortified vancomycin $(25 \mathrm{mcg} / \mathrm{mL})$ and tobramycin $(15 \mathrm{mcg} / \mathrm{mL})$. The patient continued to worsen and within two weeks developed right eye inferior corneal perforation, for which adjacent conjunctival resection, glue, and a bandage contact lens were applied (Figure 5). Topical fortified vancomycin $(25 \mathrm{mcg} / \mathrm{mL})$ and tobramycin $(15 \mathrm{mcg} / \mathrm{mL})$ were given at a lower rate of six times daily. Oral treatment consisted of doxycycline $100 \mathrm{mg}$ twice daily and $60 \mathrm{mg}$ of prednisone for one week that was tapered by $20 \mathrm{mg}$ on a weekly basis. Close follow-up showed slow improvement afterwards. After four weeks of treatment, 


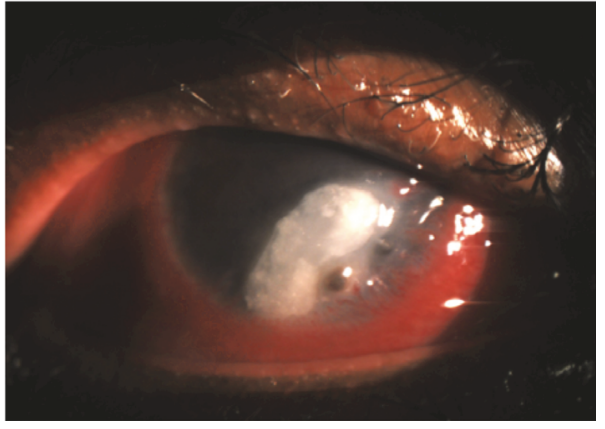

(a)

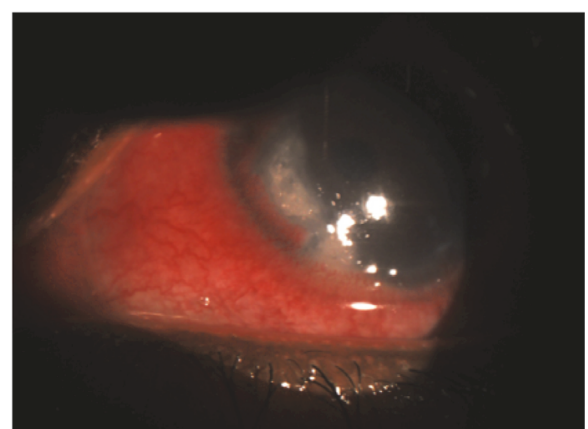

(b)

FIGURE 5: (a) Right eye slit lamp photo showing a large corneal infiltrate with a peripheral corneal perforation and pannus. (b) Left eye slit lamp photo showing peripheral ulcerative keratitis with scarring and pannus.

the corneal infiltrate resolved. The patient provided his informed consent prior to publishing this information.

\section{Discussion}

Rosacea is a chronic inflammatory skin disorder with a yet unclear pathogenesis. The diagnosis of rosacea is usually a clinical one. Flushing, centrofacial erythema, and papules are most commonly observed. In 2002, the National Rosacea Society Expert Committee developed a classification system for rosacea, describing 4 different subtypes: erythematotelangiectatic, papulopustular, phymatous, and ocular rosacea [3]. Despite different treatment approaches, the committee agrees that there is overlap between the different subtypes. In patients with skin of color it may be difficult to perceive the signs of rosacea, particularly the erythematotelangiectatic subtype.

Additionally, ocular rosacea may be easily missed as $20 \%$ develop ocular manifestations before any skin lesions. Fiftythree percent of patients present with preceding skin findings and $27 \%$ present with both simultaneously [11].

The most common ocular manifestations involve the eyelids $[14,15]$. In one report, $81 \%$ of patients presented with lid margin telangiectasia, $78 \%$ with meibomian gland dysfunction, and $65 \%$ with blepharitis [12].

Although less common, the more serious presentation involves the cornea. In one study, $33 \%$ of patients with ocular rosacea had corneal involvement [10]. The most common presentation was punctate epithelial erosions occurring in $13.6 \%[12,14]$. Wise reported that $67 \%$ of patients presenting with corneal complaints to the ophthalmology clinic had corneal neovessels and infiltrates [15].

Matrix metalloproteinases (MMP) are proinflammatory endopeptidases that have been implicated in corneal melting and stromal loss [16]. MMP-8 was found to be more elevated in the tear film of patients with rosacea compared with controls [17]. Multiple studies conducted by the Ocular Surface and Tear Center at Bascom Palmer Eye Institute looked at the concentrations of proinflammatory agents in the tear film of patients affected with rosacea and compared it to normal controls. In sum, they found that the concentrations of interleukin 1 alpha (IL-1 alpha), an inflammatory cytokine, gelatinase-B (pro-MMP-9), and MMP-9 were higher in the tear film of patients with rosacea as compared to controls $[18,19]$. In particular, the concentration of pro-MMP-3 in the tear film of patients with corneal involvement due to rosacea was higher than the concentration found in controls and patients with rosacea without corneal involvement [20].

Studies looking at other proinflammatory agents in the skin of patients affected by rosacea found that there is excessive production of both cathelicidin (LL-37) and kallikrein (KLK5), the serine protease responsible for its cleavage [2123]. In fact, LL-37 promotes inflammation, angiogenesis, and neovascularization [24]. Although not studied in the cornea of patients with rosacea, it can be hypothesized that if LL-37 was elevated in their tear film, being an angiogenic factor, it might be a contributor to corneal neovascularization. Understanding the pathogenesis of this disease may help in finding better treatment modalities.

The mainstay of treatment for blepharitis is lid hygiene [25]. Additionally, tetracycline in subantimicrobial levels has been proven to be effective in rosacea for many decades [2628]. In particular, doxycycline and its derivatives were found to improve ocular signs and symptoms of rosacea [12, 29-35]. In fact, doxycycline $100 \mathrm{mg}$ daily has been shown to improve ocular disease and increase the tear break-up time [36]. It was found to decrease the concentration of MMP-8 and MMP-9 in the tear film $[17,20]$. Topical antibiotics have been used to alter the flora of the ocular surface in addition to topical steroids [34, 37].

In patients with corneal neovascularization and stromal loss, artificial tears and gel at night, topical cyclosporine, and cautious use of steroids are recommended in addition to the above-mentioned treatment modalities $[38,39]$. Tissue adhesive or amniotic membrane or both should be applied over the cornea in the case of perforation or impending perforation [38, 40, 41]. Penetrating keratoplasty may be necessary in many severe cases $[12,41]$.

These two cases presented in the late stages of PUK due to rosacea with corneal perforation requiring interventions such as a tectonic patch graft and glue. Regardless of ethnicity, it is always important to maintain a high suspicion of rosacea in 
any individual with advanced corneal findings and negative laboratory tests.

\section{Conflicts of Interest}

The authors declare that there are no conflicts of interest regarding the publication of this paper.

\section{References}

[1] M. Berg and S. Liden, "An epidemiological study of rosacea," Acta Derm Venereol, 1989.

[2] J. K. Wilkin, "Rosacea: Pathophysiology and Treatment," Archives of Dermatology, vol. 130, no. 3, pp. 359-362, 1994.

[3] J. Wilkin, M. Dahl, M. Detmar et al., "Standard classification of rosacea: Report of the National Rosacea Society expert committee on the classification and staging of rosacea," Journal of the American Academy of Dermatology, vol. 46, no. 4, pp. 584$587,2002$.

[4] T. Rosen and M. S. Stone, "Acne rosacea in blacks," Journal of the American Academy of Dermatology, vol. 17, no. 1, pp. 70-73, 1987.

[5] A. F. Alexis, "Rosacea in patients with skin of color: Uncommon but not rare," Cutis, vol. 86, no. 2, pp. 60-62, 2010.

[6] R. M. Halder, H. L. Brooks, and V. D. Callender, "Acne in ethnic skin," Dermatologic Clinics, vol. 21, no. 4, pp. 609-615, 2003.

[7] M. Adler, "Rosacea in patients with darker skin types," Acne and Rosacea Briefs, 2002.

[8] A. Al-Dabagh, S. A. Davis, A. J. McMichael, and S. R. Feldman, "Rosacea in skin of color: Not a rare diagnosis," Dermatology Online Journal, vol. 20, no. 10, 2014.

[9] D. J. Browning and A. D. Proia, "Ocular rosacea," Survey of Ophthalmology, vol. 31, no. 3, pp. 145-158, 1986.

[10] P. A. Starr and A. Macdonald, "Oculocutaneous aspects of rosacea," in Proc R Soc Med, vol. 62, pp. 9-11, 1969.

[11] P. Borrie, "Rosacea with special reference to its ocular manifestations," British Journal of Dermatology, vol. 65, no. 12, pp. 458463, 1953.

[12] E. K. Akpek, A. Merchant, V. Pinar, and C. S. Foster, "Ocular rosacea: Patient characteristics and follow-up," Ophthalmology, vol. 104, no. 11, pp. 1863-1867, 1997.

[13] D. J. Browning, G. Rosenwasser, and M. Lugo, "Ocular rosacea in blacks," American Journal of Ophthalmology, vol. 101, no. 4, pp. 441-444, 1986.

[14] V. C. Ghanem, N. Mehra, S. Wong, and M. J. Mannis, "The prevalence of ocular signs in acne rosacea: Comparing patients from ophthalmology and dermatology clinics," Cornea, vol. 22, no. 3, pp. 230-233, 2003.

[15] G. Wise, "Ocular Rosacea ${ }^{\star *}$ From the Department of Ophthalmology, Columbia University, College of Physicians and Surgeons, and the Institute of Ophthalmology, Presbyterian Hospital, New York City." American Journal of Ophthalmology, vol. 26, no. 6, pp. 591-609, 1943.

[16] T. Sakimoto and M. Sawa, "Metalloproteinases in corneal diseases: degradation and processing," Cornea, vol. 31, no. 11, supplement 1, pp. S50-S56, 2012.

[17] M. Määttä, O. Kari, T. Tervahartiala et al., "Tear fluid levels of MMP-8 are elevated in ocular rosacea - Treatment effect of oral doxycycline," Graefe's Archive for Clinical and Experimental Ophthalmology, vol. 244, no. 8, pp. 957-962, 2006.
[18] K. Barton, D. C. Monroy, A. Nava, and S. C. Pflugfelder, "Inflammatory cytokines in the tears of patients with ocular rosacea," Ophthalmology, vol. 104, no. 11, pp. 1868-1874, 1997.

[19] A. A. Afonso, L. Sobrin, D. C. Monroy, M. Selzer, B. Lokeshwar, and S. C. Pflugfelder, "Tear fluid gelatinase B activity correlates with IL-1 $\alpha$ concentration and fluorescein clearance in ocular rosacea," Investigative Ophthalmology and Visual Science, vol. 40, no. 11, pp. 2506-2512, 1999.

[20] L. Sobrin, Z. Liu, D. C. Monroy, A. Solomon, M. G. Selzer, B. L. Lokeshwar et al., "Regulation of MMP-9 activity in human tear fluid and corneal epithelial culture supernatant," Invest Ophthalmol Vis Sci, vol. 41, pp. 1703-1709, 2000.

[21] K. Yamasaki, J. Schauber, A. Coda et al., "Kallikrein-mediated proteolysis regulates the antimicrobial effects of cathelicidins in skin," FASEB Journal, vol. 20, no. 12, pp. 2068-2080, 2006.

[22] K. Yamasaki, A. Di Nardo, A. Bardan et al., "Increased serine protease activity and cathelicidin promotes skin inflammation in rosacea," Nature Medicine, vol. 13, no. 8, pp. 975-980, 2007.

[23] A. M. Two and J. Q. Del Rosso, "Kallikrein 5-mediated inflammation in rosacea: Clinically relevant correlations with acute and chronic manifestations in rosacea and how individual treatments may provide therapeutic benefit," Journal of Clinical and Aesthetic Dermatology, vol. 7, no. 1, pp. 20-25, 2014.

[24] B. De Yang, Q. Chen, A. P. Schmidt et al., "LL-37, the neutrophil granule- and epithelial cell-derived cathelicidin, utilizes formyl peptide receptor-like 1 (FPRL1) as a receptor to chemoattract human peripheral blood neutrophils, monocytes, and T cells," Journal of Experimental Medicine, vol. 192, no. 7, pp. 1069-1074, 2000.

[25] J. E. Key, "A comparative study of eyelid cleaning regimens in chronic blepharitis," The CLAO Journal, vol. 22, no. 3, pp. 209212, 1996.

[26] I. B. Sneddon, "A clinical trial of tetracycline in rosacea," British Journal of Dermatology, vol. 78, no. 12, pp. 649-652, 1966.

[27] R. Marks, "Concepts in the pathogenesis of rosacea," British Journal of Dermatology, vol. 80, no. 3, pp. 170-177, 1968.

[28] M. T. Pelle, G. H. Crawford, and W. D. James, "Rosacea: II. Therapy," Journal of the American Academy of Dermatology, vol. 51, no. 4, pp. 499-512, 2004.

[29] A. Gupta and M. Chaudhry, "Rosacea and its management: an overview," Journal of the European Academy of Dermatology and Venereology, vol. 19, no. 3, pp. 273-285, 2005.

[30] V. J. Marmion, "Tetracyclines in the treatment of ocular rosacea," Proc R Soc Med, vol. 62, pp. 11-12, 1969.

[31] A. G. Knight and C. F. Vickers, "A follow-up of tetracyclinetreated rosacea. With special reference to rosacea keratitis," $\mathrm{Br} J$ Dermatol, vol. 93, pp. 577-580, 1975.

[32] J. Frucht-Pery, E. Sagi, I. Hemo, and P. Ever-Hadani, "Efficacy of doxycycline and tetracycline in ocular rosacea," American Journal of Ophthalmology, vol. 116, no. 1, pp. 88-92, 1993.

[33] J. Frucht-Pery, A. S. Chayet, S. T. Feldman, S. Lin, and S. I. Brown, "The effect of doxycycline on ocular rosacea," American Journal of Ophthalmology, vol. 107, no. 4, pp. 434-435, 1989.

[34] R. Odom, M. Dahl, J. Dover et al., "Standard management options for Rosacea, part 2: Options according to subtype," Cutis, vol. 84, no. 2, pp. 97-104, 2009.

[35] D. U. Stone and J. Chodosh, "Oral Tetracyclines for Ocular Rosacea: An Evidence-Based Review of the Literature," Cornea, vol. 23, no. 1, pp. 106-109, 2004.

[36] M. J. Quarterman, D. W. Johnson, D. C. Abele, J. L. Lesher Jr., D. S. Hull, and L. S. Davis, "Ocular rosacea: Signs, symptoms, 
and tear studies before and after treatment with doxycycline," Archives of Dermatology, vol. 133, no. 1, pp. 49-54, 1997.

[37] W. Zamil and K. Arfaj, "Spontaneous corneal perforation in ocular rosacea," Middle East African Journal of Ophthalmology, vol. 17, no. 2, p. 186, 2010.

[38] İ. K. Müftüoğlu and Y. A. Akova, "Clinical findings, followup and treatment results in patients with ocular rosacea," Turk Oftalmoloiji Dergisi, vol. 46, no. 1, pp. 1-6, 2016.

[39] A. Arman, D. D. Demirseren, and T. Takmaz, "Treatment of ocular rosacea: comparative study of topical cyclosporine and oral doxycycline," Int J Ophthalmol, vol. 8, pp. 544-549, 2015.

[40] A. K. Jain and J. Sukhija, "Aminiotic membrane transplantation in ocular rosacea," Annals of Ophthalmology, vol. 39, no. 1, pp. 71-73, 2007.

[41] G. López-Valverde, E. Garcia-Martin, J. Larrosa-Povés, V. PoloLlorens, and L. Pablo-Júlvez, "Therapeutical Management for Ocular Rosacea," Case Reports in Ophthalmology, vol. 7, no. 1, pp. 237-242, 2016. 


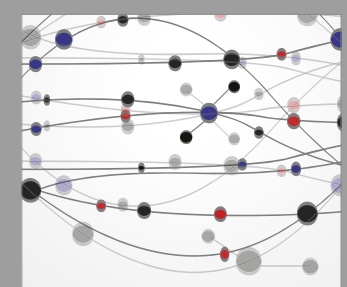

The Scientific World Journal
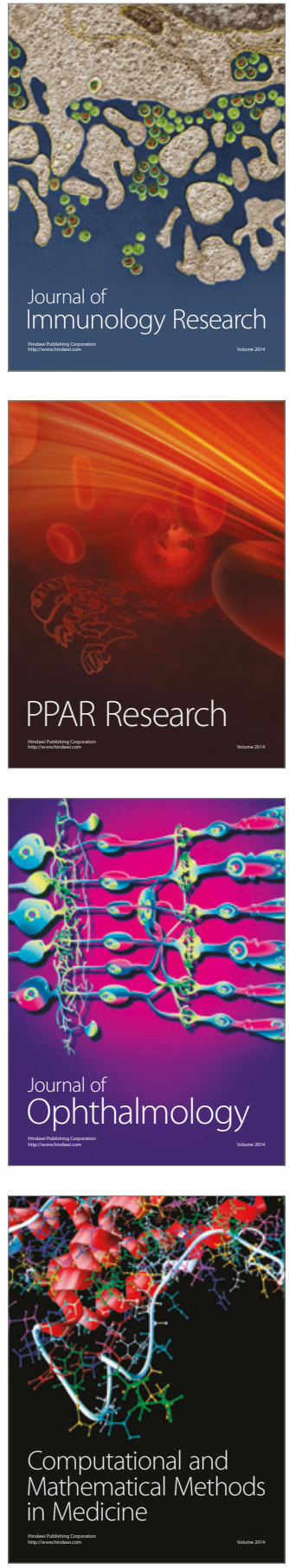

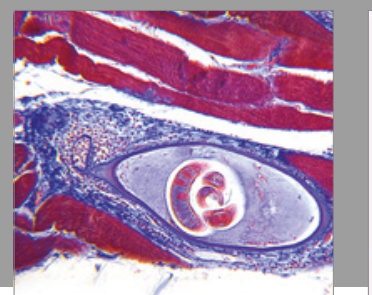

Gastroenterology Research and Practice
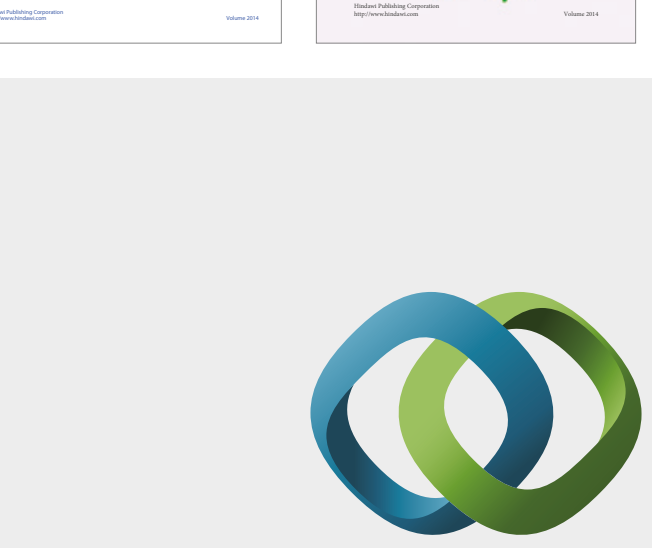

\section{Hindawi}

Submit your manuscripts at

https://www.hindawi.com
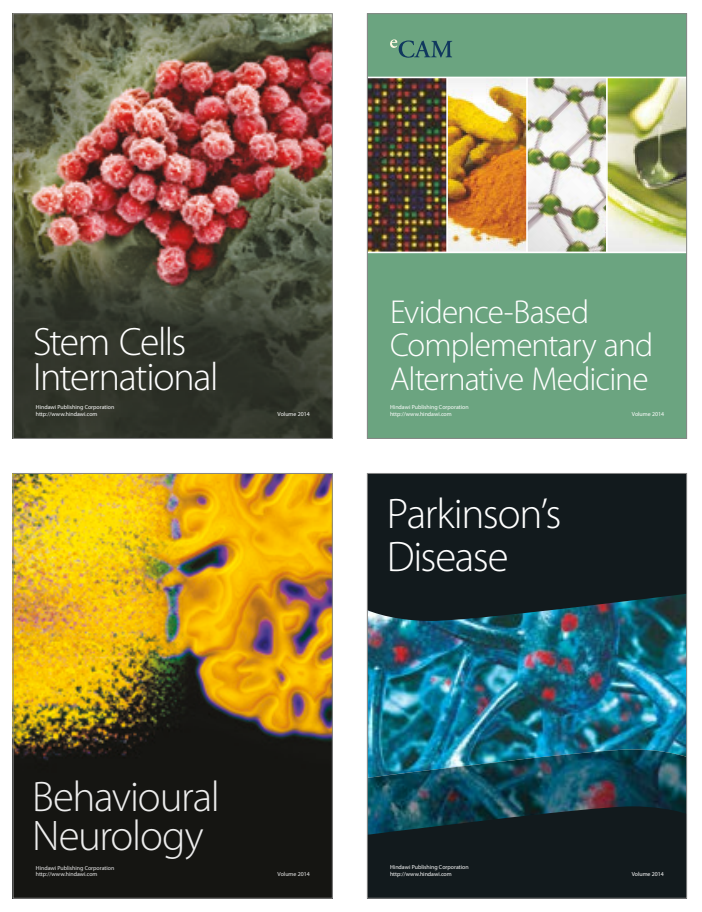
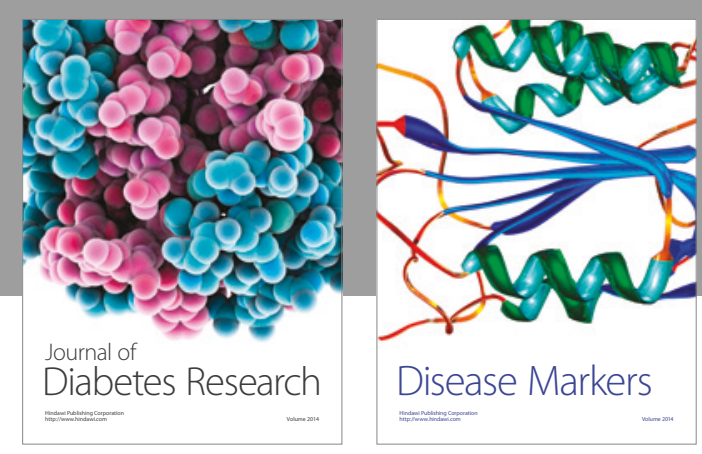

Disease Markers
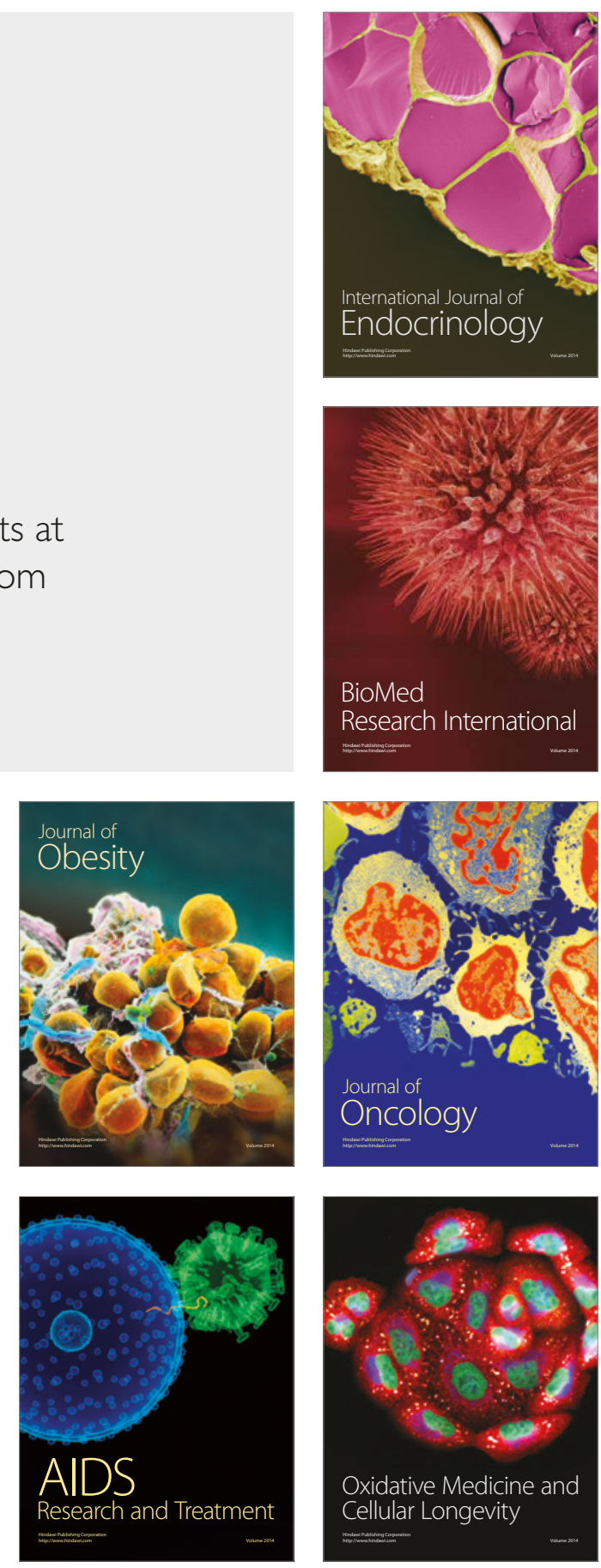